\title{
Investigating the Interaction of Cellulose Nanofibers Derived from Cotton with a Sophisticated 3D Human Lung Cell Coculture
}

\author{
Martin J. D. Clift, ${ }^{\dagger}$ E. Johan Foster, ${ }^{\dagger}$ Dimitri Vanhecke, ${ }^{\ddagger}$ Daniel Studer, ${ }^{\ddagger}$ Peter Wick, ${ }^{\S}$ Peter Gehr, ${ }^{\ddagger}$ \\ Barbara Rothen-Rutishauser, ${ }^{*,+}$ and Christoph Weder, ${ }^{*+}$ \\ ${ }^{+}$Adolphe Merkle Institute and Fribourg Center for Nanomaterials, University of Fribourg, Rte de l'Ancienne Papeterie, \\ CH-1723 Marly, Switzerland \\ ${ }^{\ddagger}$ Institute of Anatomy, University of Bern, Baltzerstrasse 2, CH-3000, Bern 9, Switzerland \\ ${ }^{\S}$ Empa, Swiss Federal Laboratories for Materials Science and Technology, Materials-Biology Interactions Laboratory, Lerchenfeldstrasse \\ 5, CH-9014, St. Gallen, Switzerland
}

\begin{abstract}
Cellulose nanofibers are an attractive component of a broad range of nanomaterials. Their intriguing mechanical properties and low cost, as well as the renewable nature of cellulose make them an appealing alternative to carbon nanotubes (CNTs), which may pose a considerable health risk when inhaled. Little is known, however, concerning the potential toxicity of aerosolized cellulose nanofibers. Using a 3D in vitro triple cell coculture model of the human epithelial airway barrier, it was observed that cellulose nanofibers isolated from cotton $(\mathrm{CCN})$ elicited a significantly $(p<0.05)$ lower cyto-
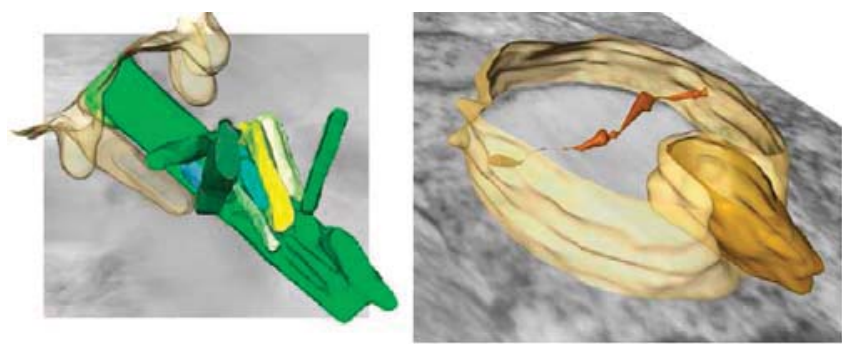
toxicity and (pro-)inflammatory response than multiwalled CNTs (MWCNTs) and crocidolite asbestos fibers (CAFs). Electron tomography analysis also revealed that the intracellular localization of CCNs is different from that of both MWCNTs and CAFs, indicating fundamental differences between each different nanofibre type in their interaction with the human lung cell coculture. Thus, the data shown in the present study highlights that not only the length and stiffness determine the potential detrimental (biological) effects of any nanofiber, but that the material used can significantly affect nanofiber-cell interactions.
\end{abstract}

\section{INTRODUCTION}

Due to their exceedingly high strength and stiffness, cellulose nanowhiskers isolated from biosources, such as wood, straw, and cotton, have emerged as an attractive component of a broad range of advanced materials. ${ }^{1,2}$ Examples of such include highperformance polymer nanocomposites, ${ }^{4}$ mechanically adaptive materials, ${ }^{5}$ and mesoporous photonic solids. ${ }^{6}$ The mechanical properties, low cost, and renewable nature of cellulose nanowhiskers make them an appealing alternative to other nanofibers (defined as a nano-object (a material with one, two or three external dimensions in the nanoscale $(1-100 \mathrm{~nm})))^{3}$ with two similar external dimensions in the nanoscale and the third dimension significantly larger), such as multiwalled carbon nanotubes (MWCNTs), which were recently reported to cause adverse effects in vitro and in vivo, such as DNA damage, ${ }^{7}$ increased inflammatory cytokine/chemokine expression and oxidative stress. ${ }^{8}$ MWCNTs have further been suggested to elicit asbestos-like effects, such as an increased inflammatory granulamatous formation on the peritoneal aspect of the diaphragm in vivo. ${ }^{9}$ Indeed, MWCNTs share several characteristics with asbestos, including a high aspect (length to width) ratio and high stiffness. ${ }^{10}$ Recently, it was suggested that the progressing carcinogenic effects observed for MWCNTs could be triggered by these features ${ }^{9}$ and not their chemical composition (specifically their Fe content), which had previously been suggested on the basis of in vitro and in vivo studies. ${ }^{11}$ Because cellulose nanowhiskers also exhibit a high aspect ratio and high stiffness, ${ }^{1}$ it is imperative to ask the question if they could also cause similar undesirable biological effects. The lung, when also considering ingestion, injection, or application of nano-objects to the skin, is the primary portal of entry to the human body, and thus, nanoobjects pose the greatest potential human health risk to this organ. Cellulose nanowhiskers may be inhaled in an aerosolized form during various stages of the lifecycle, ${ }^{12}$ in particular, when handled in an individualized form (i.e., during isolation and processing), upon extraction from nanocomposites or aerogels under high-wear conditions (e.g., processing such as cutting or sanding, which has been reported as a problem in the case of asbestos ${ }^{10}$ ), or upon release from the matrix after disposal (i.e., matrix degradation). It is therefore necessary to assess the potential risks associated with these nanofibers and the nanomaterials (defined as "a material that has one or more external dimension in the nanoscale or which is nanostructured") $)^{3}$ made from cellulose nanowhiskers before they can be broadly exploited. $^{13}$ 

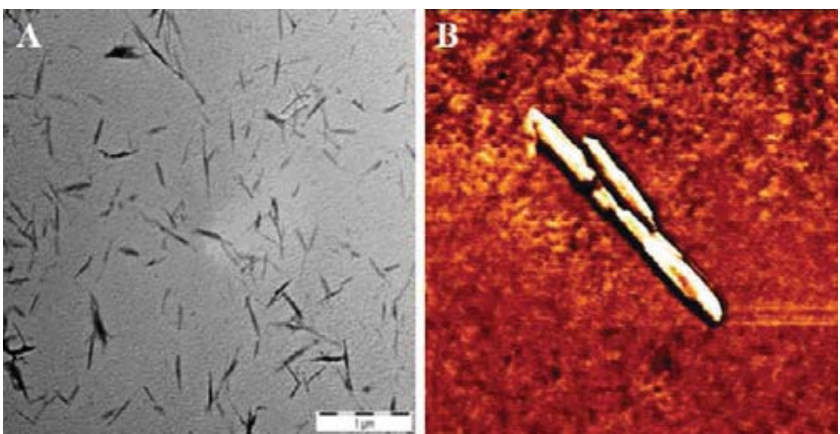

Figure 1. (A) Typical morphology of the cellulose nanowhiskers derived from cotton (CCNs) as imaged by transmission electron microscopy. (B) Shows individual CCNs as imaged by atomic force microscopy on mica $(650 \times 650 \mathrm{~nm})$.

Experimental data regarding interactions between cellulose nanofibers and lung cells are severely limited. Recently, Moreira et al. ${ }^{14}$ conducted an in vitro study to explore the potential genotoxicity and cell proliferation of bacterial cellulose secreted from Gluconacetobacter xylinus. This type of cellulose assembles into several micrometer long ribbons that form a dense reticulated network and, thus, exhibits a structure that is quite different from that of the individualized nanowhiskers studied here (Figure 1A,B). The material did not affect cell viability (as assessed by the MTT colorimetric assay) and induced no genotoxicity in $\mathrm{CHO}$ cells or 3T3 fibroblast cells (as determined by the comet assay and the Salmonella tryphimurium reversion assay (Ames test)) up to a concentration of $0.1 \mathrm{mg} \cdot \mathrm{mL}^{-1}$. The appropriateness of the latter test to assess the potential genotoxicity of nano-objects to humans is, however, of current debate, because the interaction of nano-objects with bacteria and mammalian cells are different. ${ }^{15}$ In addition, no effect upon cell viability was observed with bacterial cellulose when probed using the MTT colorimetric assay. ${ }^{14}$ Several papers have also discussed the toxicity of cellulose fibers with diameters of $>100 \mathrm{~nm} .{ }^{16}$ These studies showed that cellulose fibers elicit a significantly lower inflammatory response, as well as fewer granulomas and reduced mesothelioma formation compared to asbestos fibers in vivo, ${ }^{16}$ while in vitro, the same fibers showed no significant lactate dehydrogenase (LDH) release from A549 epithelial cells. Therefore, the results from the available toxicity studies, ${ }^{14,16}$ the fact that cellulose is biodegradable, and the notion that the biosources from which cellulose nanowhiskers are derived are deemed "safe", may suggest that cellulose nanowhiskers are indeed benign. Nevertheless, the dimensions and structure of individualized cellulose nanowhiskers $(\sim 10-30 \times \sim 100-2000 \mathrm{~nm}$, depending upon the source) are vastly different from those of the microfibrillated cellulose (entangled fibers with a length of several micrometers) that was studied before. ${ }^{1,14}$ Furthermore, cellulose degradation occurs primarily through enzymatic processes, which normally does not occur in the human body. One must also consider that surface-modified cellulose nanowhiskers are frequently used for nanocomposite fabrication. ${ }^{1}$ The extremely high surface area to weight ratio suggests that the surface (and not bulk) chemistry may determine the rate of hydrolytic degradation processes and how such nanowhiskers present themselves to cells. This therefore supports the notion that the potential hazard associated with nano-objects can be strongly associated with their surface area and reactivity. ${ }^{17}$ Thus, an understanding of how cellulose nanowhiskers interact with biological systems is imperative prior to their application.
Table 1. Characterization Data for Cellulose Nanowhiskers Derived from Cotton (CCNs), Multiwalled Carbon Nanotubes (MWCNTs), and Crocidolite Asbestos Fibers (CAFs) in Cell Culture Media (RPMI 1640 Containing 5\% Human Serum, 1\% L-Glutamine, and $1 \%$ Penicillin/Streptomycin $)^{a}$

$\begin{array}{llll}\text { (nano)fiber type } & \text { CCNs } & \text { MWCNTs } & \text { CAFs } \\ \text { length }(\mu \mathrm{m}) & 0.22 \pm 0.067 & 1-10^{*} & 3 \rightarrow 50 \S^{*} \\ \text { diameter }(\mathrm{nm}) & 15 \pm 5 & 5-30^{*} & 100^{*} \\ \text { aspect ratio (diameter/length) } & \sim 15 & 200-250 & 40-250 \\ \text { chemical content (\%wt) } & \mathrm{NA} & \mathrm{Fe}(0.05)^{*} & \mathrm{Fe}(15.1)^{*} \\ & & \mathrm{Mg}(0.01)^{*} & \mathrm{Mn}(0.09)^{*} \\ & \mathrm{Ni}(0.12)^{*} & \mathrm{Cr}(0.002)^{*} \\ & \mathrm{Co}(<0.001)^{*} & \mathrm{Ni}(0.001)^{*} \\ & & \mathrm{Co}(0.001)^{*}\end{array}$

$a^{*}$ indicates data summarized from an analysis previously published in refs 22, 23, and 25; NA refers to not applicable; § refers to percentage of CAFs (by number); Length $(\mu \mathrm{m}) 3-5$ (53.8\%), 5-10 (36\%), 10-25 $(9.2 \%), 25-50(1.0 \%)$, and $>50(0.1 \%) .^{22,23,25}$

\section{EXPERIMENTAL SECTION}

Materials and Methods. Chemicals and Reagents. All chemicals and reagents were purchased from Sigma-Aldrich, Switzerland, unless otherwise stated.

Isolation of Cotton Cellulose Nanowhiskers (CCNs). Cellulose nanowhiskers were isolated from cotton (CCNs) using the previously reported protocol, ${ }^{1}$ which largely follows the procedure of Dong et al. ${ }^{18}$ Briefly, $5.2 \mathrm{~g}$ of Whatman No. 1 filter paper was combined with $250 \mathrm{~mL}$ of deionized water and then blended at high speed for $5 \mathrm{~min}$. The resulting pulp was cooled at $4{ }^{\circ} \mathrm{C}$ in an ice bath, and a total of $140 \mathrm{~mL}$ of concentrated sulfuric acid (98\%) was then slowly added under vigorous stirring over a period of $45 \mathrm{~min}$. The addition rate of the sulfuric acid was controlled to keep the temperature below $30^{\circ} \mathrm{C}$. After all the sulfuric acid was added to the sample, the suspension was heated to $50{ }^{\circ} \mathrm{C}$ and stirred at this temperature for $4.5 \mathrm{~h}$. The dispersion was then cooled to room temperature and centrifuged for $15 \mathrm{~min}$ at $3400 \mathrm{rpm}$, and the supernatant solution was decanted. The remaining mixture was subsequently dispersed in $100 \mathrm{~mL}$ of $\mathrm{H}_{2} \mathrm{O}$ by shaking and subsequently centrifuged as before. The process was repeated 5 times until the supernatant reached $\mathrm{pH} 4$. The resulting solid was then dialyzed over $48 \mathrm{~h}$ in $\mathrm{H}_{2} \mathrm{O}$ (with 5 changes of water). The resulting slurry was then sonicated for $3 \mathrm{~h}$ and incubated at room temperature overnight. The dispersed supernatant, containing the CCNs, was then separated from any solids by decanting. The concentration of the dispersion was finally determined $\left(10 \mathrm{mg} \cdot \mathrm{mL}^{-1}\right)$ by vacuum drying a $5 \mathrm{~mL}$ sample and weighing the remaining solid.

Characteristics of CCNs. Sulfuric acid hydrolysis of Whatman filter paper yields CCNs with a typical width of $15 \pm 5 \mathrm{~nm}$, a length of $220 \pm$ $67 \mathrm{~nm}$, and an average aspect ratio of $\sim 15$ (Figure 1A,B and Table 1). The hydrolysis protocol employed introduces a small amount, relative to overall $\mathrm{OH}$ content $\left(\sim 31 \mathrm{mmol} \cdot \mathrm{kg}^{-1}\right)$, of sulfate groups, as determined by titration, on the surface of the nanowhiskers. ${ }^{19}$

Atomic Force Microscopy. CCNs were deposited using $50 \mu \mathrm{L}$ of a $0.1 \mathrm{mg} \cdot \mathrm{mL}^{-1}$ aqueous stock solution that was placed on a freshly cleaved mica surface. The solvent was evaporated at $60^{\circ} \mathrm{C}$ under vacuum for 24 h. A JPK SPM Control Station III with a NanoWizard II standalone atomic force microscope (AFM) head was then used to acquire images in tapping mode using silicon cantilevers (NANO WORLD, TESPA-50). All micrographs are presented in top-view with no filtering to ensure that all images were produced with the same quality.

Transmission Electron Microscopy. Dispersion and dimensions of the cellulose nanowhiskers were studied using transmission electron 
microscopy (TEM; CM100, Philips Electron Optics, Zurich, Switzerland) operating at an accelerating voltage of $80 \mathrm{kV}$. A $10 \mu \mathrm{L}$ drop of a $0.1 \mathrm{mg} \cdot \mathrm{mL}^{-1}$ stock solution was placed on a copper grid, supported by $3 \mathrm{~nm}$ carbon with a layer of $50 \mathrm{~nm}$ polymer film below. The samples were dried at $60^{\circ} \mathrm{C}$ in a vacuum oven for $2 \mathrm{~h}$ and then imaged. Dimensions quoted (Table 1) are averages of $10-15$ whiskers from five random TEM images.

Monocultures. To determine how CCNs interacted with each different cell type of the 3D in vitro coculture, all biochemical end points were also assessed for each cell type contained within the coculture model. Human monocyte derived macrophages (MDM) and dendritic cells (MDDC) (MDDC were obtained by incubating monocytes with the growth factors GM-CSF and IL-4) were isolated from human whole blood and cultured at $37^{\circ} \mathrm{C}, 5 \% \mathrm{CO}_{2}$ as previously described in. ${ }^{20}$ The human bronchial epithelial cell-line 16HBE14o-, kindly donated by Dr. D. Gruenert (Cardiovascular Research Institute, University of California, San Francisco), were cultured as described in ref 21.

3D Triple Cell Coculture Model of the Epithelial Airway Barrier. Monocultures of MDM, MDDC, and 16HBE14o- cells were combined on 12 well trans-well inserts ( area $=0.9 \mathrm{~cm}^{2}$ ), as described in 20 and 21 .

Preparation and Exposure of CCNs to Cell Cultures. A stock dispersion of $2 \mathrm{mg} \cdot \mathrm{mL}^{-1} \mathrm{CCNs}$ in $\mathrm{H}_{2} \mathrm{O}$ was prepared by diluting the original dispersion (vide supra) with water. The stock solution was then thoroughly vortexed for up to $1 \mathrm{~min}$ and diluted to concentrations of 0.005 , 0.015 , and $0.03 \mathrm{mg} \cdot \mathrm{mL}^{-1}$ in specific cell culture media (either epithelial cell media or complete cell media). The working concentrations were then exposed to the different cell cultures (either monocultures or the triple cell coculture), as a suspension, for $24 \mathrm{~h}$ at $37^{\circ} \mathrm{C}, 5 \% \mathrm{CO}_{2}$.

Preparation and Exposure of MWCNTS and CAFs to Cell Cultures. MWCNTs and CAFs were employed within the present study as positive control (nano)fibers. MWCNTs (Cheaptubes) were dispersed in Pluronic F127, as previously described in ref 22, to provide a stable and well-dispersed sample. In ref 28 and with subsequent studies, it has been observed that when Pluronic F127 is used at 160 ppm to disperse these MWCNTs that no adverse effects are induced in vitro. ${ }^{22,23} \mathrm{CAFs}$ (a kind donation from the National Research Institute of Occupational Diseases (Johannesburg, South Africa) were weighed out and suspended directly in cell culture media. Samples were then sonicated in a water bath for $10 \mathrm{~min}$. Stock solutions of both MWCNTs and CAFs were then diluted to form both a low and high working concentration of 0.005 and $0.03 \mathrm{mg} \cdot \mathrm{mL}^{-1}$ respectively. The working concentrations for both MWCNTs and CAFs were exposed, as a suspension, to either monocultures or the triple cell coculture for $24 \mathrm{~h}$ at $37^{\circ} \mathrm{C}, 5 \% \mathrm{CO}_{2}$.

Biochemical Analysis. Measurement of Cytotoxicity. The potential for CCNs, MWCNTs, and CAFs to induce cytotoxicity in the four different cell cultures was assessed by the level of lactate dehydrogenase $(\mathrm{LDH})$ present in the cell supernatants, as previously described by ref 24 . LDH is a cytosolic enzyme that can be released by cells following the permeabilization of the cell membrane. LDH is thus released into the cell medium due to membrane leakage. Membrane leakage can cause subsequent inflammatory reactions and can also enable the passage of NPs through the epithelial (or epithelialtype (i.e., trophoblast)) layer. To assess the total LDH release from the different cell cultures used, $0.2 \%$ Triton $\mathrm{X} 100$ was used as a positive control. All LDH results are presented as a percentage of the total LDH release from cells. The ability of the CCNs, MWCNTs, and CAFs to adsorb to the surface of the LDH enzyme, eliciting a false negative toxicity, was also investigated. No significant LDH adsorption was observed (data not presented).

Measurement of (Pro-)Inflammatory Cytokine/Chemokine Stimulation. The ability for CCNs, MWCNTs, and CAFs to stimulate the proinflammatory cytokine tumor necrosis factor (TNF)- $\alpha$ (MDM and MDDC monocultures, as well as the triple cell coculture) and the inflammatory chemokine interleukin (IL)-8 (16HBE14o- monocultures and triple cell coculture) was assessed using enzyme-linked immunosorbant (ELISA) diagnostic kits (R\&D Systems, Switzerland). TNF- $\alpha$ is mainly produced by macrophages as well as dendritic cells and controls a wide range of biological reactions, such as the induction of additional cytokines involved in the innate immune response. IL-8 is also a mediator of the innate immune response and serves as a chemotactic factor for granulocytes. IL-8 is released by epithelial cells and many other different cells. The ability of the CCNs, MWCNTs, and CAFs to adsorb to the surface of either the TNF- $\alpha$ or IL- 8 protein, eliciting a false negative toxicity, was also investigated. No significant TNF- $\alpha$ or IL- 8 protein adsorption was observed (data not presented).

Electron Tomography. In conventional electron microscopy, only projections in two dimensions of a thin three-dimensional body are imaged. In such a case, the cross-section of, for example, a fiber can be a bar, an ellipse, or a circle. These representations of the fiber could be addressed to other two- or three-dimensional forms (i.e., spheres, cuboids, or discs). Electron tomography, however, shows the defined three-dimensional structure of any investigated body. Furthermore, a large nano-object can hide a smaller one in conventional electron microscopy. The tilting method employed by electron tomography, however, enables the small, hidden nano-object to become visible. Ultrathin sections were cut $300 \mathrm{~nm}$ thick and mounted onto 100 mesh copper grids. The samples were screened for a suitable area with a CM12 TEM (FEI Co. Philips Electron Optics, Zurich, Switzerland). Tomography imaging was then recorded on a selected area with a Tecnai F20 TEM (FEI, Eindhoven, The Netherlands) equipped with a GIF Tridiem energy filter and Ultrascan 1000 CCD camera (Gatan, Pleasanton, U.S.A.). The tomography was recorded at a magnification of $34000 \times$, respectively, performing a continuous tilt angle shift from $\max -70^{\circ}$ to $+70^{\circ}$ with a dual tilt Fischione specimen holder (Fischione Instruments, U.S.A.). To correct for the missing wedge $\left(-90^{\circ}\right.$ until $-70^{\circ} /+90^{\circ}$ until $+70^{\circ}$ ) dual tilt axis acquisition was performed with an angle difference of $90^{\circ}$. Image processing and $3 \mathrm{D}$ stack reconstruction was performed with the Inspect 3D software V.3.0. (FEI Company). Further image processing and the reconstruction of the different (nano)fibers was performed using AMIRA 5.2.2 (Visage Imaging, Berlin, Germany). ${ }^{25}$

Data and Statistical Analysis. All results are presented as the mean \pm standard error of the mean (SEM). All data was found to be normally distributed (data not shown). Subsequent statistical significance analysis was performed using a parametric one-way analysis of variance (ANOVA), followed by a Tukey's pairwise comparisons post hoc test when appropriate (MINITAB, version 15.1, MINITAB Inc., 2006). Results were considered significant if $p \leq 0.05$.

\section{RESULTS AND DISCUSSION}

The use of a sophisticated three-dimensional in vitro triple cell coculture model of the human epithelial airway barrier ${ }^{20,26}$ allowed investigation, for the first time, of the interactions of cellulose nanowhiskers with human lung cells. The triple cell coculture model consists of primary, monocyte derived macrophages (MDM; apical layer) and dendritic cells (MDDC; basolateral layer) derived from human whole blood, as well as an epithelial layer formed by the human bronchial epithelial cellline $16 \mathrm{HBE} 140-{ }^{21}$ It has been shown that this in vitro model mimics the precise structure of this important aspect of the airway wall in the human lung as it is in vivo. ${ }^{26}$ In addition to this, the triple cell coculture has been used as a basis for studying how different types of nano-objects may interact with the important immune and barrier cells of the epithelial airway barrier. Fluorescent latex, nontoxic micrometer-sized and nanosized spherical nano-objects have been used to study the entry mechanism and intracellular fate that these micrometer/nano-objects may take 

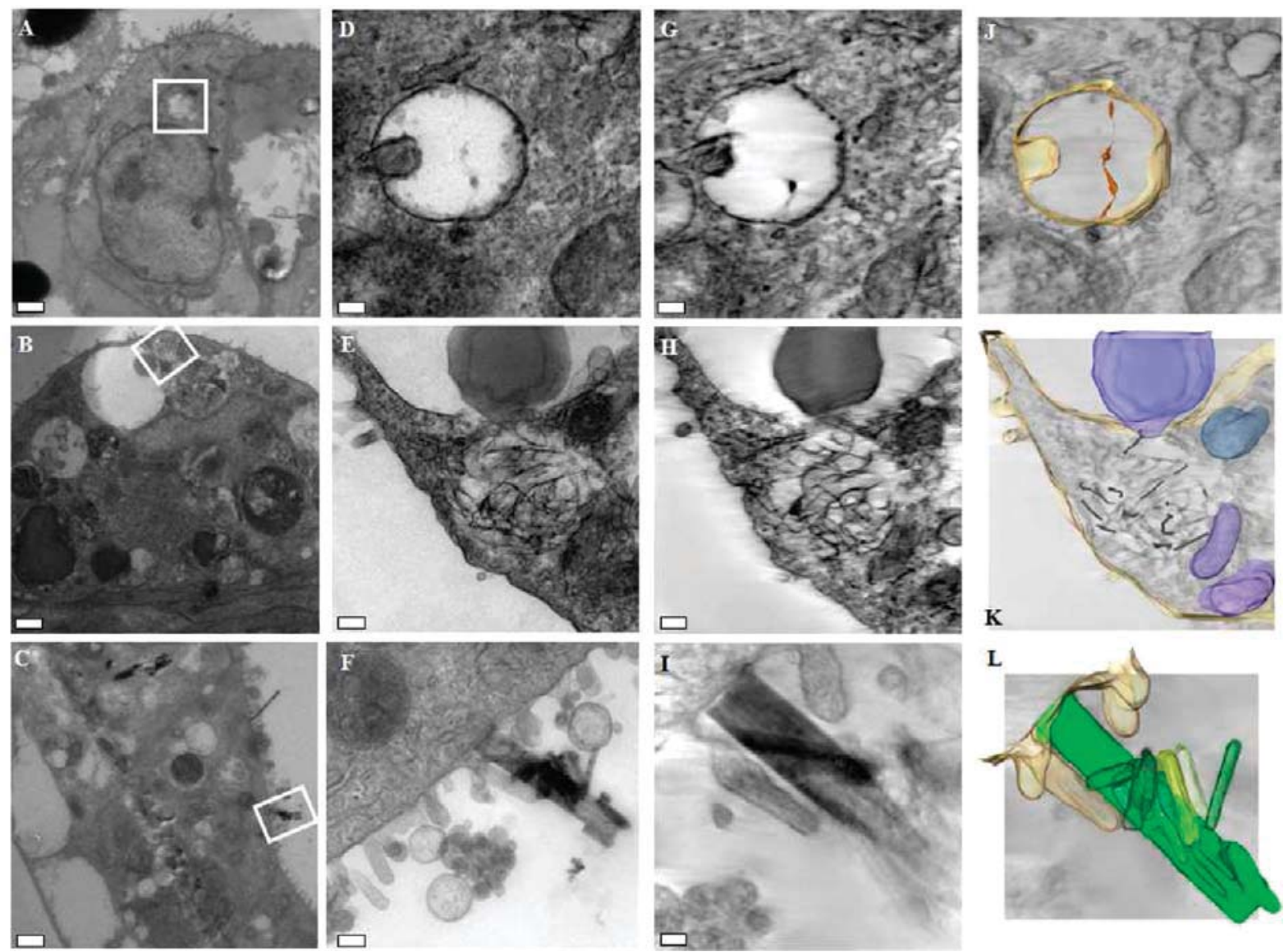

Figure 2. Electron tomography 2D still images of the triple cell coculture system after exposure to (A) cellulose nanowhiskers derived from cotton (CCNs), (B) multiwalled carbon nanotubes (MWCNTs), and (C) crocidolite asbestos fibers (CAFs) after $24 \mathrm{~h}$ submerged culture exposure at $0.03 \mathrm{mg} \cdot \mathrm{mL}^{-1}$ in a controlled environment of $37^{\circ} \mathrm{C}, 5 \% \mathrm{CO}_{2}$. The CCNs (A) are observed to be located within a vesicle inside the human monocyte derived macrophages (MDM) on the apical layer of the in vitro triple cell coculture model. MWCNTs (B) are also observed inside a vesicle, while CAFs (C) elicit the classical response known as "frustrated phagocytosis" by the MDM. The white scale bar in A-C represents $1.0 \mu \mathrm{m}$. Images D, E, and F are the projected image of the inset (white square) in images A, B, and C respectively. Images G-I show representative tomographic slices of images D-F. The white scale bar in images $\mathrm{D}-\mathrm{I}$ represents $0.2 \mu \mathrm{m}$. Images J-L show the 3D reconstructed (rendered) electron tomograms of images $\mathrm{D}-\mathrm{F}$, as produced from AMIRA 5.2.2. CCNs (J) are highlighted as an orange/brown color, while the MWCNTs (K; please note that only a selected number of MWCNTs were chosen for complete rendering) and CAFs (L) are colored gray and green, respectively. The vesicular body is always a transparent yellow color $(\mathrm{J}-\mathrm{L})$. Also, highlighted in the rendered tomographic image of the MWCNTs (K) are intracellular bodies (vesicles) in blue/purple.

following suspension exposure. ${ }^{21}$ In addition, $\mathrm{Fe}_{2} \mathrm{O}_{3}$ and $\mathrm{Au}$ core nano-objects with an ATTO polymer shell, ${ }^{27}$ as well as "naked" (i.e., no shell) citrate stabilized $\mathrm{Au}$ NPs and $\mathrm{Au}$ with a polyethylene glycol polymer shell $\mathrm{NPs}^{28}$ have been shown to localize within lysosomes, but not the mitochondria, when exposed to the triple cell coculture model by either aerosol or suspension methods. Numerous studies have also investigated the potential adverse effects that nano-objects may elicit following their interaction with the epithelial airway barrier in vitro. In a study by Brandenberger and colleagues, ${ }^{29}$ Au nano-objects were assessed for their ability to affect cell signaling, including proinflammatory pathways, and induce cytotoxicity. Although the model was shown to be sensitive to lipopolysaccharide exposure, no significant $(p>0.05)$ synergistic or suppressive effects were observed over a $24 \mathrm{~h}$ period. In contrast, however, a study in which different nanofiber (single-walled CNTs (SWCNTs)) and nano-objects (titanium dioxide $\left(\mathrm{TiO}_{2}\right)$ ) were assessed for their ability to stimulate pro-inflammatory cytokine/chemokine markers, as well as affect total antioxidant capacity (TAC) within the triple cell coculture. ${ }^{30}$ It was reported that the in vitro model elicited a significant decrease $(p<0.05)$ in the TAC following SWCNTs exposure, while $\mathrm{TiO}_{2}$ caused a significant increase $(p<0.05)$ in TAC of the triple cell coculture. Similar results were found for the pro-inflammatory cytokine TNF- $\alpha$ and chemokine IL- 8 , in contrast to the findings of ref 29 . These findings highlight that the triple cell coculture model responds differently to a variety of nano-objects. Due to this knowledge and the plethora of information already gathered in relation to lung cell nano-object interactions in vitro using this model, the triple cell coculture was employed in the current study to explore the lung cell interaction of cellulose nanowhiskers isolated from cotton by hydrolysis with sulfuric acid (cotton cellulose nanowhiskers, CCNs). These particular cellulose nanowhiskers contain a small concentration of negatively charged sulfate groups on their surface, which are a result from the hydrolysis protocol and contribute considerably to their colloidal stability in water. They were selected for this study because they are the type most commonly used by researchers (Figure 1A,B and Table 1). ${ }^{1}$

Electron tomography images of the triple cell coculture system after exposure to CCNs revealed that these particles interacted 

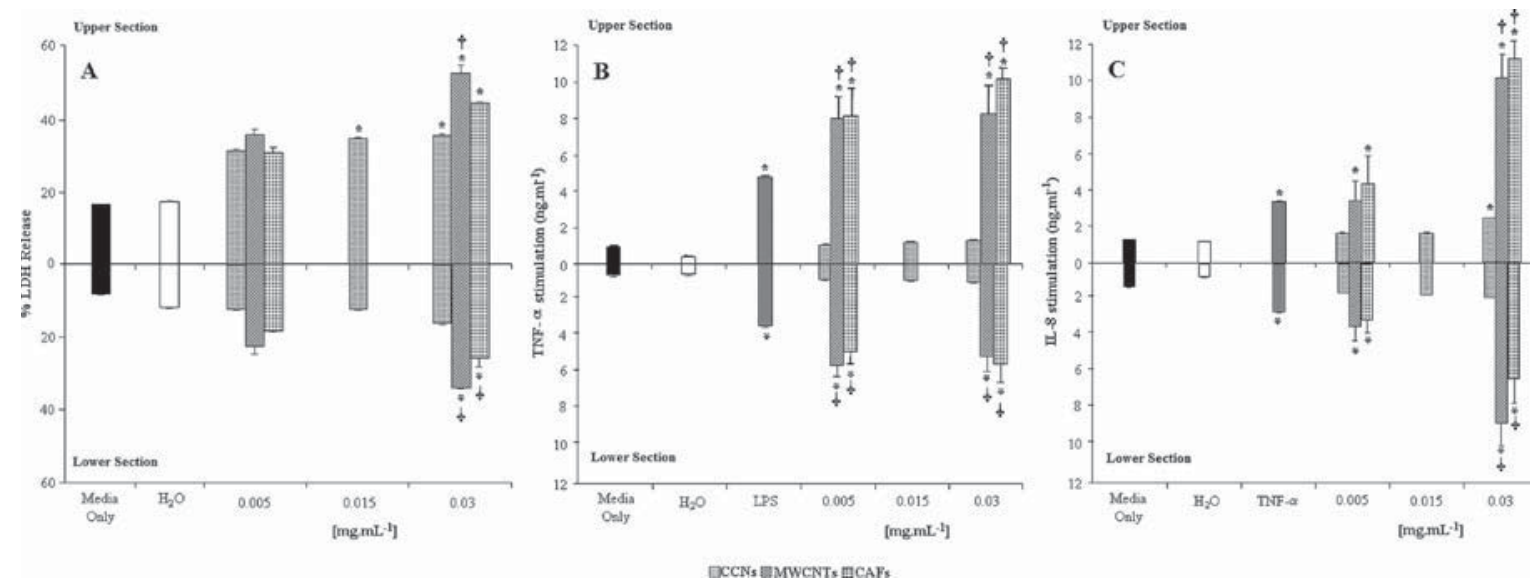

Figure 3. (A) Cytotoxicity (lactate dehydrogenase (LDH) release compared to total LDH release caused by $0.2 \%$ Triton X100 exposure), (B) tumor necrosis factor (TNF)- $\alpha$ stimulation, and (C) interleukin (IL)-8 stimulation of cellulose nanowhiskers derived from cotton (CCNs), multiwalled carbon nanotubes (MWCNTs), and crocidolite asbestos fibers (CAFs) in the apical layer (consisting of human blood monocyte derived macrophages (MDM) and an epithelial layer of the 16HBE14o- human bronchial cell-line) and the basolateral layer (consisting of human blood monocyte derived dendritic cells (MDDC)) of the 3D triple cell coculture model of the epithelial airway barrier. The apical aspect of the triple cell coculture was exposed to each (nano)fiber type for $24 \mathrm{~h}$ at concentrations of 0.005 and $0.03 \mathrm{mg} \cdot \mathrm{mL}^{-1}$ and in case of CCNs also $0.015 \mathrm{mg} \cdot \mathrm{mL}^{-1}$ at $37^{\circ} \mathrm{C}$ in an atmosphere with $5 \%$ $\mathrm{CO}_{2}$. The effects of $\mathrm{H}_{2} \mathrm{O}$ were also assessed since this was the buffer in which the CCNs were originally suspended. In both (B) lipopolysaccharide (LPS) and (C) TNF- $\alpha$ were used as positive assay controls at $1 \mathrm{mg} \cdot \mathrm{mL}^{-1}$. Data is presented as mean \pm standard error of the mean (SEM) with $n=3$. ${ }^{*}$ Represents a significant difference $(p<0.05)$ compared to the negative control (medium only). ${ }^{\dagger}$ Represents a significant difference $(p<0.05)$ compared to CCNs at the same concentration.

only with the MDM on the apical side of the triple cell coculture (Figure 2). No CCNs were observed within the epithelial cell layer or the MDDC after $24 \mathrm{~h}$ of exposure to a suspension comprising of the CCNs at a concentration of $0.03 \mathrm{mg} \cdot \mathrm{mL}^{-1}$ in a controlled environment $\left(37^{\circ} \mathrm{C}, 5 \% \mathrm{CO}_{2}\right)$. Similar results were also observed for both the MWCNTs and crocidolite asbestos fibers (CAFs) under the same experimental conditions. The observation that none of the (nano)fibers used in the present study underwent trans-epithelial translocation supports the findings of Mercer et al., ${ }^{31}$ who reported that MWCNTs only interacted with macrophages in the alveolar epithelium of C57BL/6J mice (56 days after a dose of $80 \mu \mathrm{g}$ had been administered by pharyngeal aspiration). The fate of MWCNTs in the current study, which were observed to be "bundled", showed localization within vesicles, similar to the interaction of spherical nano-objects with cells, ${ }^{32}$ while CAFs showed classical signs of frustrated phagocytosis. ${ }^{33}$ Interestingly, the CCNs did not cause any form of frustrated phagocytosis of the macrophage cells. Instead, they were completely internalized within a vesicle, suggesting that they enter these phagocytic cells through a form of endocytosis. The precise mechanism of this process is subject to further investigation. The observation that the CCNs, and likewise the MWCNTs, do not induce frustrated phagocytosis can be attributed to their relatively short length. Alternatively, it may be related to the specific surface chemistry of the CCNs. Thus, chemical surface modification, as it is practiced to compatibilize nanowhiskers with polymer matrices for nanocomposite fabrication ${ }^{1}$ could dramatically change interactions of the nanowhiskers with mammalian cells. This concept is not novel, since it has been shown that the surface chemistry of spherical nanoobjects can dictate the specific response of cells in vivo and in vitro. ${ }^{34}$ Nonetheless, it is necessary to assess this further for the safe handling and use of cellulose nanowhiskers when considering their potential use and applications. In addition, the role of protein interaction and exchange on the surface of CCNs should be examined, because this is known to significantly affect the interaction of different nano-objects with mammalian cells. ${ }^{35}$

The manner in which nano-objects interact with cells in vitro has been shown to significantly affect the subsequent cellular response. ${ }^{34}$ Our investigation of the cellulose nanowhiskers' ability to cause cellular damage (cytotoxicity) by the release of the cytosolic enzyme lactate dehydrogenase (LDH) showed that at a low CCN concentration $\left(0.005 \mathrm{mg} \cdot \mathrm{mL}^{-1}\right)$ no significant $(p>0.05)$ cytotoxicity occurred after $24 \mathrm{~h}$ of exposure on the apical layer of the triple cell coculture model (Figure 3A). Similarly, no significant $(p>0.05)$ cytotoxicity was observed on the basolateral side, up to a CCN concentration of 0.03 $\mathrm{mg} \cdot \mathrm{mL}^{-1}$. This finding is consistent with previous data on the toxicity of cellulose fibers with a diameter of $>100 \mathrm{~nm}$ (i.e., nonnano-objects), ${ }^{16}$ as well as those on bacterial cellulose. ${ }^{14}$ The latter showed no alteration to the viability of $\mathrm{CHO}$ or $3 \mathrm{~T} 3$ fibroblast cells when treated with cellulose dispersions at concentrations of up to $0.1 \mathrm{mg} \cdot \mathrm{mL}^{-1}$ for $72 \mathrm{~h}$. A significant $(p<$ 0.05 ) dose-dependent toxicity was, however, observed for CCN concentrations of 0.015 and $0.03 \mathrm{mg} \cdot \mathrm{mL}^{-1}$ on the apical side of the triple cell coculture model. No adsorption of the LDH enzyme with the CCNs, MWCNTs, or CAFs was observed for any of the concentrations explored. These results clearly show that despite the limited cytotoxicity observed, CCNs can elicit a cytotoxic response in vitro at concentrations that are deemed realistic. ${ }^{10}$ Interestingly, however, at the highest concentration tested, both MWCNTs and CAFs elicited a much higher LDH release from the apical layer (combined of both MDM and epithelial cells) compared to the CCNs. Although the CAFs were found to elicit $20 \%$ more LDH at the same concentration, only the MWCNTs were observed to be significantly $(p<0.05)$ cytotoxic compared to the CCNs when exposed for $24 \mathrm{~h}$ at a concentration of $0.03 \mathrm{mg} \cdot \mathrm{mL}^{-1}$ (causing a $31 \%$ increase in the $\mathrm{LDH}$ released from the cells in the apical layer). It is important to highlight that single-walled CNTs (SWCNTs) were not investigated within the 

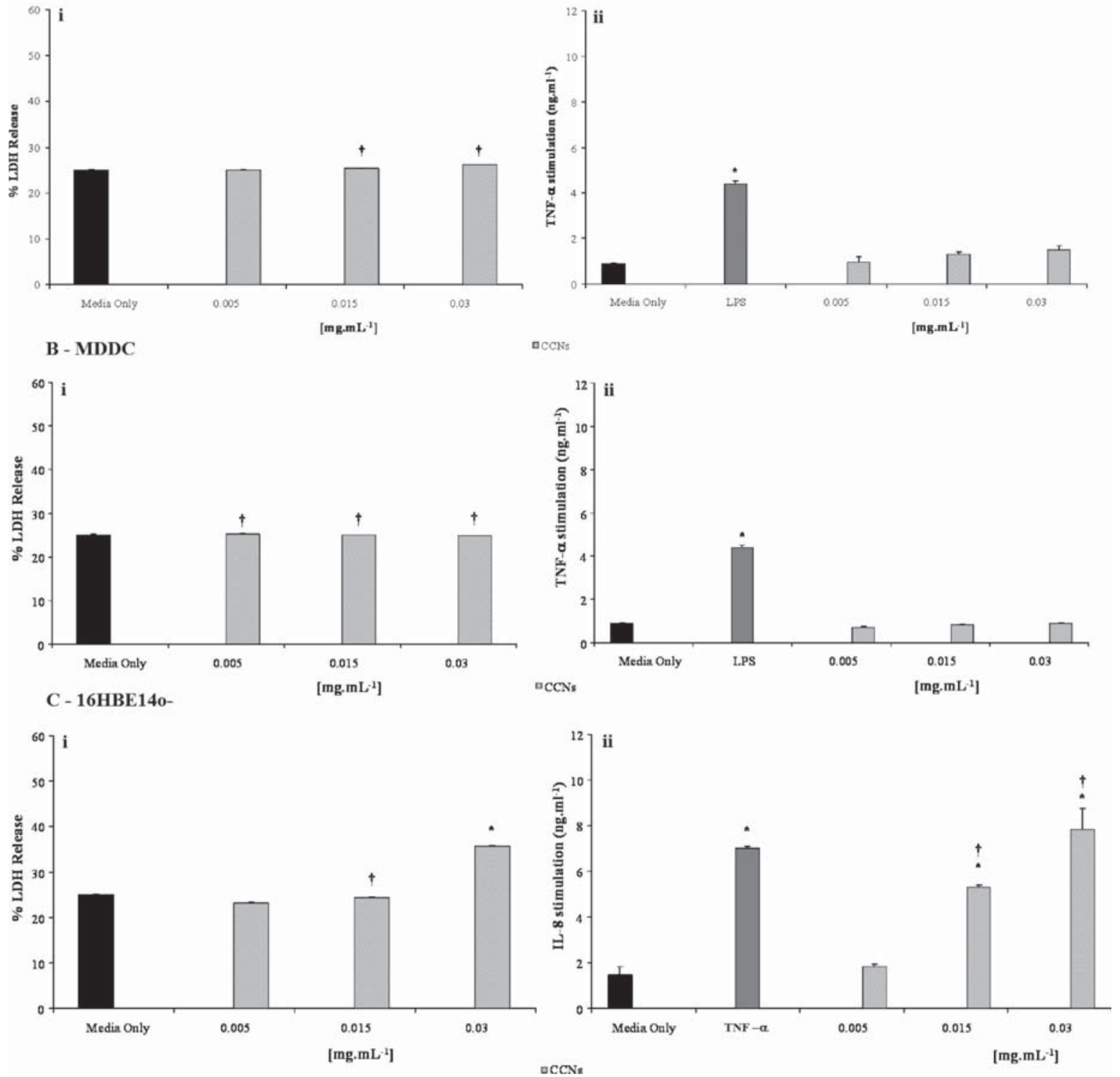

Figure 4. (A; i) Cytotoxicity (lactate dehydrogenase (LDH) release) and (ii) tumor necrosis factor (TNF)- $\alpha$ stimulation in MDM, (B; i) cytotoxicity (lactate dehydrogenase (LDH) release) and (ii) tumor necrosis factor (TNF)- $\alpha$ stimulation in MDDC, (C; i) cytotoxicity (lactate dehydrogenase $(\mathrm{LDH})$ release) and (ii) interleukin (IL)-8 stimulation in 16HBE14o- monocultures following exposure of cellulose nanowhiskers derived from cotton $(\mathrm{CCNs})$ in suspension for $24 \mathrm{~h}$ at concentrations of $0.005,0.015$, and $0.03 \mathrm{mg} \cdot \mathrm{mL}^{-1}$ at $37^{\circ} \mathrm{C}$ in an atmosphere with $5 \% \mathrm{CO}_{2}$. In both $(\mathrm{A})$ and $(\mathrm{B})$ lipopolysaccharide (LPS) and (C) TNF- $\alpha$ were used as positive assay controls at $1 \mathrm{mg} \cdot \mathrm{mL}^{-1}$. Data is presented as mean \pm standard error of the mean (SEM) with $n=3 .{ }^{*}$ Represents a significant difference $(p<0.05)$ compared to the negative control (medium only). ${ }^{\dagger}$ Represents a significant difference $(p<0.05)$ compared to CCNs at the same concentration following exposure to the $3 \mathrm{D}$ in vitro triple cell coculture model (Figure 3 ).

current study. In the past few years it has been widely reported that MWCNTs and not SWCNTs can elicit and induce adverse effects in vivo and in vitro. ${ }^{7}$ Furthermore, it has been shown that, depending upon the specific production process and physicochemical characteristics of SWCNTs, that they are liable to enzyme degradation when interacting with biological systems, which can lessen the inflammatory potential of these nanofibers. ${ }^{36}$ Thus, suggesting that SWCNTs can change their structural characteristics and interact with biological systems as nano-objects and not specifically nanofibers. In addition to this, cellulose nanowhiskers are primarily intended for application within construction materials, such as use in mechanical reinforcement. ${ }^{2}$ Therefore, due to the increased strength and durability of
MWCNTs renders them a more specific comparison than SWCNTs. Due to their ability to induce relatively low adverse biological effects, ${ }^{7}$ however, and in-line with the data presented in the current study, a comparison between cellulose nanowhiskers and SWCNTs would be of interest to further understand how the specific material may contribute to the interaction of nanofibers with biological systems.

Investigation using electron microscopy (Figure 2) showed that despite this significant increase in LDH release, cells did not show any form of significant membrane damage, rounding, or cellular bodies associated with different mechanisms of cell death. Studies that probe this aspect further are ongoing. The effects of such xenobiotic exposure to the basolateral side of the triple cell 
coculture model showed that both the MWCNTs and CAFs caused a significant $\mathrm{LDH}$ release compared to both the negative control and CCNs (concentration of $0.03 \mathrm{mg} \cdot \mathrm{mL}^{-1}$ ) after $24 \mathrm{~h}$. It is significant that the CCNs did not induce cytotoxicity on the basolateral layer (composed of MDDC) of the triple cell coculture at any of the concentrations tested. This suggests that the cellular interplay does indeed differ between the various (nano)fibers investigated and that the immune protector cells (i.e., macrophages) are able to cope with CCNs better than both MWCNTs and CAFs.

This difference is further reflected by the different (pro)inflammatory reactions of the triple cell coculture when exposed to the (nano)fibers studied. Figure $3 \mathrm{~B}$ shows the release of the pro-inflammatory cytokine tumor necrosis factor- $\alpha$ (TNF- $\alpha$ ) into the media. In both the apical and basolateral media chambers, the CCNs caused no significant $(p>0.05)$ stimulation of TNF- $\alpha$ in comparison to the baseline values (i.e., the cell culture media only). By contrast, both MWCNTs and CAFs elicited significant $(p<0.05)$ TNF- $\alpha$ release in both chambers of the triple cell coculture model. Similar observations were also made for the stimulation of the inflammatory chemokine interleukin-8 (IL-8) by CCNs, MWCNTs, and CAFs (Figure 3C). While a dose-dependent increase in IL-8 release from the cells was observed for MWCNTs and CAFs, a significant increase ( $p<0.05$; relative to baseline levels) in the production of this inflammatory chemokine was observed after CCN exposure at $0.03 \mathrm{mg} \cdot \mathrm{mL}^{-1}$ (apical layer only). The IL- 8 response in vitro has not been previously assessed for CCNs. Because this inflammatory chemokine response simulates that of the response observed with TNF- $\alpha$, it is therefore possible that the CCNs do not interfere with immune-system pathways in vitro and provides further evidence that the macrophages are able to adequately cope with CCN exposure with limited negative responses. Further in-depth research is required to fully understand this behavior. In addition, the (pro)-inflammatory response observed for CCNs further supports previous studies, ${ }^{16}$ which showed that larger cellulose fibers $(>100 \mathrm{~nm}$ in diameter) elicited a significantly lower "inflammatory" response (as measured by the stimulation of TNF- $\alpha$ both in vitro and in vivo) than CAFs. Interestingly, the (pro)-inflammatory response upon MWCNTs exposure is similar to that of CAFs, providing further evidence that MWCNTs can stimulate a similar cellular response in vitro to asbestos fibers.

Finally, it is important to highlight the effects observed from the triple cell coculture following exposure to CCNs, MWCNTs, and CAFs are different to the effects of the same (nano)fibers upon the monoculture of each cell within the epithelial airway model (Figure 4). Although in vitro monocultures provide the basis for high-throughput analysis for nanotoxicology, they do not however, represent a realistic model of how nano-objects will interact with a specific organ of the body. Recently, there have been increased efforts to establish more realistic models to study the toxic potential of nano-objects, such as the triple cell coculture system employed within the present study. As previously highlighted, this model provides a clear basis for investigating the interaction of nano-objects with the lung. ${ }^{20,26}$ Figure 4 shows the effects of CCNs on MDM, MDDC, and $16 \mathrm{HBE} 140-$ epithelial cell monocultures using the same end points as presented in relation to their effects on the triple cell coculture (Figure 3). It was observed that in each different monoculture (MDM, MDDC, and 16HBE14o- cells), the LDH release was significantly $(p<0.05)$ different to that found following CCN exposure to the triple cell coculture. This was also evident in the level of IL- 8 stimulation in $16 \mathrm{HBE} 14 \mathrm{o}$ - cells at both 0.015 and $0.03 \mathrm{mg} \cdot \mathrm{mL}^{-1}(p<0.05)$. Interestingly, however, no significant differences $(p>0.05)$ between the triple cell coculture and monocultures (MDM and MDDC) were observed in regards to the TNF- $\alpha$ response after $\mathrm{CCN}$ exposure. This is in contrast to the findings of ref 24 who reported a significant difference $(p<0.05)$ in the TNF- $\alpha$ response elicited by the triple cell coculture compared to monocultures following SWCNT and $\mathrm{TiO}_{2}$ exposure. The reason for this observation for the MDM monocultures in the present study, could be due to the direct interaction with the specifically exposed (nano)fibers (the characteristics of the nanofibers may also significantly contribute the results presented, when considering previous observations ${ }^{30}$ ), as also seen with the triple cell coculture model (Figure 2). The similar effects observed between the TNF- $\alpha$ stimulation in the MDDC monocultures and triple cell coculture, however, are more interesting. It could be that the cellular interplay and signaling that occurs within the triple cell coculture mimics that of a direct exposure of the nanofibers, or it could simply be a specific end-point result, since similar results were not observed between these two different cell cultures in regards to $\mathrm{LDH}$ release. In summary therefore, the findings of the present study (monocultures vs triple cell coculture responses) highlight that (i) monoculture analysis for the screening of potential adverse effects of NPs is not sufficient, as it can express false negative or positive results and can provide information that is not reliable as a comparison to the in vivo response and (ii) the differences observed between the triple cell coculture are both end point and nano-object specific.

\section{CONCLUSION}

In conclusion, the presented data clearly shows that cellulose nanowhiskers isolated from cotton, when delivered as a suspension to a reliable in vitro triple cell coculture of the epithelial airway barrier containing human lung cells, elicit a dose-dependent cytotoxicity and (pro)-inflammatory response. However, the reactions triggered upon exposure to $\mathrm{CCNs}$ were significantly lower than the responses caused by both MWCNTs and CAFs. In addition, the reported results comparing the triple cell coculture with monocultures, clearly shows that the effects of each different exposure upon each individual monoculture was significantly different from those observed for the triple cell coculture. This finding is consistent with previous research that has shown similar results with both diesel exhaust particles and manufactured nano-objects, including CNTs and $\mathrm{TiO}_{2}{ }^{30}$ and implies that the use of monoculture in vitro systems is not appropriate. Meaningful screening of the potential hazards posed by nano-objects should rely on more sophisticated in vitro models that are specific to the organ of primary interest and provide a well-structured and characterized version that mimics the in vivo situation. ${ }^{26}$ While further, in-depth toxicological analysis is ongoing to assess the potential for cellulose nanowhiskers to cause oxidative stress, stimulate (pro-)inflammatory cytokines/chemokines and genotoxicity, the initial data reported in the present study reveal that in spite of the supposed biocompatibility of cellulose, CCNs can elicit adverse effects in vitro. Gratifyingly, however, these effects are significantly less pronounced than those caused by both MWCNTs and CAFs, suggesting that the material used can significantly affect nanofibre-cell interactions. It is imperative that great care is taken 
when generalizing our findings to other cellulose nanowhisker types, because surface chemistry, surface charges, as well as the length, aspect ratio, and stiffness vary considerably and are likely to influence interactions with mammalian cells.

\section{AUTHOR INFORMATION}

\section{Corresponding Author}

*E-mail: barbara.rothen@unifr.ch; christoph.weder@unifr.ch.

\section{Notes}

Conflict of Interest. The authors have no conflict of interest. The authors are entirely responsible for the data collection and writing of this manuscript.

\section{ACKNOWLEDGMENT}

The authors thank Barbara Tschirren for technical assistance with the cell culture work and acknowledge financial support from the European Respiratory Society (Fellowship LTRFMC1572-2010 to Dr. M. J. D. Clift), the Swiss National Science Foundation (National Research Programme 64 \#4064-131264 and Division 3 \#3100A0_118420), the German Research Foundation (DFG SPP 1313), the Animal Free Research Foundation, the Doerenkamp-Zbinden Foundation, Dr. Alfred Bretscher for the funding of the Tecnai F20 TEM, and the Adolphe Merkle Foundation.

\section{REFERENCES}

(1) Habibi, Y.; Lucia, L. A.; Rojas, O. J. Chem. Rev. 2010, 110, 3479-3500.

(2) Eichhorn, S. J.; Dufresne, A.; Aranguren, M.; Marcovich, N. E.; Capadona, J. R.; Rowan, S. J.; Weder, C.; Thielemans, W.; Roman, M.; Renneckar, S.; Gindl, W.; Veigel, S.; Keckes, J.; Yano, H.; Abe, K.; Nogi, M.; Nakagaito, A. N.; Mangalam, A.; Simonsen, J.; Benight, A. S.; Bismarck, A.; Berglund, L. A.; Peijs, T. J. Mater. Sci. 2010, 45 (1), 1-33.

(3) International Organization for Standardization (ISO) Nanotechnologies-Terminology and definitions for nano-objects-Nanoparticle, nanofiber, and nanoplate. Technical Specification (ISO/TS) 27687:2008; First published 8/15/2008.

(4) Capadona, J. R.; Van Den Berg, O.; Capadona, L. A.; Schroeter, M.; Rowan, S. J.; Tyler, D. J.; Weder, C. Nat. Nanotechnol. 2007, 2, 765-769.

(5) Capadona, J. R.; Shanmuganathan, K.; Tyler, D. J.; Rowan, S. J.; Weder, C. Science 2008, 319, 1370-1374.

(6) Shopsowitz, K. E.; Qi, H.; Hamad, W. Y.; MacLachlan, M. J. Nature 2010, 468, 422-425.

(7) Johnston, H. J.; Hutchison, G. R.; Christensen, F. M.; Peters, S.; Hankin, S.; Aschberger, K.; Stone, V. Nanotoxicology 2010, 4, 207-246.

(8) Rothen-Rutishauser, B.; Brown, D. M.; Piallier-Boyles, M.; Kinloch, I. A.; Windle, A. H.; Gehr, P.; Stone, V. Nanotoxicology 2010, 4, 331-342.

(9) Poland, C. A.; Duffin, R.; Kinloch, I.; Maynard, A.; Wallace, W. A. H.; Seaton, A.; Stone, V.; Brown, S.; MacNee, W.; Donaldson, K. Nat. Nanotechnol. 2008, 3, 423-428.

(10) Donaldson, K.; Murphy, F. A.; Duffin, R.; Poland, C. A. Part. Fibre Toxicol. 2010, 7, 5.

(11) Kagan, V. E.; Tyurina, Y. Y.; Tyurin, V. A.; Konduru, N. V.; Potapovich, A. I.; Osipov, A. N.; Kisin, E. R.; Schwegler-Berry, D.; Mercer, R.; Castranova, V.; Shvedova, A. A. Toxicol. Lett. 2006, 165, 88-100.

(12) Hansen, F. S.; Maynard, A.; Baun, A.; Tickner, J. A. Nat. Nanotechnol. 2008, 3, 444-447.

(13) Kovacs, T.; Naish, V.; O'Connor, B.; Blaise, C.; Gagné, F.; Hall, L.; Trudeau, V.; Martel, P. Nanotoxicology 2010, 4, 255-270.
(14) Moreira, S.; Silva, N. B.; Almeida-Lima, J.; Rocha, H. A. O.; Medeiros, S. R. B.; Alves, C., Jr; Gama, F. M. Toxicol. Lett. 2009, $189,235-241$.

(15) Claxton, L. D.; Umbuzeiro, G.; de, A.; DeMarini, D. M. Environ. Health Perspect. 2010, 118, 1515-1522.

(16) Cullen, R. T.; Searl, A.; Miller, B. G.; Davis, J. M. G.; Jones, A. D. J. Appl. Toxicol. 2000, 20, 49-60.

(17) Duffin, R.; Tran, L.; Brown, D.; Stone, V.; Donaldson, K. Inhal. Toxicol. 2007, 19, 849-856.

(18) Dong, X. M.; Kimura, T.; Revol, J. F.; Gray, D. G. Langmuir 1996, 12, 2076-2082.

(19) Shanmuganathan, K.; Capadona, J. R.; Rowan, S. J.; Weder, C. J. Mater. Chem. 2010, 20, 180-186.

(20) Rothen-Rutishauser, B. M.; Kiama, S. G.; Gehr, P. Am. J. Respir. Cell Mol. Biol. 2005, 32, 281-289.

(21) Blank, F.; Rothen-Rutishauser, B.; Gehr, P. Am. J. Respir. Cell Mol. Biol. 2007, 36, 669-677.

(22) Wick, P.; Manser, P.; Limbach, L. K.; Dettlaff-Weglikowska, U.; Krumreich, F.; Roht, S.; Stark, W. J.; Bruinink, A. Toxicol. Lett. 2007, $168,121-131$.

(23) Thurnherr, T.; Su, D. S.; Diener, L.; Weinberg, G.; Manser, P.; Pfaender, N.; Arrigo, R.; Schuster, M. E.; Wick, P.; Krug, H. F. Nanotoxicology 2009, 3, 319-338.

(24) Brown, D. M.; Wilson, M. R.; MacNee, W.; Stone, V.; Donaldson, K. Toxiciol. Appl. Pharm. 2001, 175, 191-199.

(25) Thurnherr, T.; Brandenberger, C.; Fischer, K.; Diener, L.; Manser, P.; Maeder-Althaus, X.; Kaiser, J. P.; Krug, H. F.; RothenRutishauser, B.; Wick, P. Toxicol. Lett. 2011, 200, 176-186.

(26) Rothen-Rutishauser, B.; Blank, F.; Muehlfeld, Ch.; Gehr, P. Exp. Opin. Drug Metabol. Toxicol. 2008, 4, 1075-1089.

(27) Lehmann, A. D.; Parak, W. J.; Zhang, F.; Zulqurnain, A.; Rocker, C.; Nienhaus, G. U.; Gehr, P.; Rothen-Rutishauser, B. Small 2010, 6, 753-761.

(28) Brandenberger, C.; Muhlfeld, C.; Zulqurnain, A.; Lenz, A.-G.; Schmid, O.; Parak, W.; Gehr, P.; Rothen-Rutishauser, B. Small 2010a, 6, 1669-1678.

(29) Brandenberger, C.; Rothen-Rutishauser, B.; Muhlfeld, C.; Schmid, O.; Ferron, G. A.; Maier, K. L.; Gehr, P.; Lenz, A.-G. Toxicol. Appl. Pharmacol. 2010b, 242, 56-65.

(30) Mueller, L.; Riediker, M.; Wick, P.; Mohr, M.; Gehr, P.; RothenRutishauser, B. J. R. Soc. Interface 2010, 7, S27-S40.

(31) Mercer, R. R.; Hubbs, A. F.; Scabilloni, J. F.; Wang, L.; Battelli, L. A.; Schwegler-Berry, D.; Castranova, V.; Porter, D. W. Part. Fibre Toxicol. 2010, 7, 28.

(32) Geiser, M.; Rothen-Rutishauser, B.; Kapp, N.; Schurch, S.; Kreyling, W.; Schulz, H.; Semmler, M.; Hof, V. I.; Heyder, J.; Gehr, P. Environ. Health Perspect. 2005, 113, 1555-1560.

(33) Brown, D. M.; Kinloch, I. A.; Bangert, U.; Windle, A. H.; Walter, D. M.; Walker, G. S.; Scotchford, C. A.; Donaldson, K.; Stone, V. Carbon 2007, 45, 1743-1756.

(34) Unfried, K.; Albrecht, C.; Klotz, L. O.; von Mikecz, A.; GretherBeck, S.; Schins, R. P. F. Nanotoxicology 2007, 1, 1-20.

(35) Monopoli, M. P.; Walczyk, D.; Campbell, A.; Elia, G.; Lynch, I.; Bombelli, F. B.; Dawson, K. A. J. Am. Chem. Soc. 2011, 133, 2525-2534.

(36) Kagan, V. E.; Konduru, N. V.; Feng, W.; Allen, B. L.; Conroy, J.; Volkov, Y.; Vlasova, I. I.; Belikova, N. A.; Yanamala, N.; Kapralov, A.; Tyurina, Y. Y.; Shi, J.; Kisin, E. R.; Murray, A. R.; Franks, J.; Stolz, D.; Gou, P.; Klein-Seetharaman, J.; Fadeel, B.; Star, A.; Shvedova, A. A. Nat. Nanotechnol. 2010, 5, 354-359. 